\title{
Tensions in Antibiotic Prescribing
}

\section{Pitting Social Concerns Against the Interests of Individual Patients}

\author{
Joshua P. Metlay, MD, PhD, Judy A. Shea, PhD, Linda B. Crossette, MPH, \\ David A. Asch, MD, MBA
}

BACKGROUND: To reduce the prevalence of antibioticresistant bacteria in the community, physicians must optimize their use of antibiotics. However, optimal use from the perspective of the community (reserving newer agents for future use) is not always consistent with optimal use from the perspective of the individual patient (prescribing newer, broader agents).

OBJECTIVES: To identify preferred patterns of antibiotic prescribing for patients with community-acquired pneumonia (CAP), measure explicit attitudes toward antibiotics and antibiotic resistance, and determine the relationship between these prescribing patterns and attitudes.

DESIGN: Cross-sectional anonymous mail survey.

PARTICIPANTS: National random sample of 400 generalist physicians (general internal medicine and family practice) and 429 infectious diseases specialists.

MEASUREMENTS: Rank ordering of antibiotic preferences for a hypothetical outpatient with CAP and reasons for antibiotic selection. Endorsement of attitudes regarding antibiotic prescribing decisions and resistance.

RESULTS: Both generalists and infectious diseases specialists were more likely to prefer newer, broader drugs for the treatment of CAP compared to older agents still recommended by national guidelines. Physicians rated the issue of contributing to antibiotic resistance lowest among 7 determinants of their choices.

CONCLUSIONS: Despite national guidelines and increasing public awareness, the public health concern of contributing to the problem of antibiotic resistance does not exert a strong

Received from the Philadelphia Veterans Affairs Medical Center (JPM, DAA), and the Division of General Internal Medicine, Department of Medicine (JPM, JAS, DAA), the Center for Clinical Epidemiology and Biostatistics (JPM, JAS, LBC, DAA), the Center for Education and Research on Therapeutics (JPM, $D A A)$, and the Leonard Davis Institute of Health Economics (JPM, JAS, DAA), University of Pennsylvania, Philadelphia, Pa.

Portions of these study results were presented at the national meeting of the Society of General Internal Medicine, May 2-5, 2001, San Diego, Calif.

Address correspondence and requests for reprints to Dr. Metlay: University of Pennsylvania School of Medicine, 712 Blockley Hall, 423 Guardian Dr., Philadelphia, PA 19104 (e-mail: jmetlay@cceb.med.upenn.edu). impact on physician prescribing decisions for CAP. Future efforts to optimize antibiotic prescribing decisions will need to consider options for increasing the impact of public health issues on the patient-oriented decisions of individual physicians.

KEY WORDS: antibiotics; drug utilization; physician's practice patterns; drug resistance, microbial; pneumonia.

J GEN INTERN MED 2002;17:87-94.

A ntimicrobial drug use is the selective force driving the emergence of drug-resistant bacterial pathogens in community settings. ${ }^{1,2}$ The recent rise in resistance among common pathogens, such as S. pneumoniae and E. coli, has threatened traditional approaches to the empiric management of many community-acquired infections. ${ }^{3,4}$ As a result, tremendous attention has been placed on reducing the emergence of drug resistance by changing the antibiotic prescribing practices of community-based physicians. ${ }^{5}$

One target of this attention is the prescription of antimicrobial drugs for conditions for which antibacterial therapy is of no proven benefit, particularly nonbacterial infections such as upper respiratory infections, the common cold, and acute bronchitis., ${ }^{6,7}$ Targeting such inappropriate practices is not controversial. However, even when antimicrobial use is appropriate, the choice of particular agents may still promote the development of resistance. ${ }^{8}$ In fact, there is increasing evidence that patterns of antibiotic use in outpatient settings have a strong impact on patterns of emerging resistance, ${ }^{9-11}$ and therefore the issue of the societal impact of antibiotic prescribing decisions is a relevant factor in defining optimal patterns of antibiotic use in the outpatient setting.

For example, many potential agents effectively treat community-acquired pneumonia (CAP). Recent guidelines endorse several antibiotic choices as equally appropriate for the treatment of outpatients with CAP, including macrolides, fluoroquinolones, and tetracyclines. ${ }^{3,12}$ However, some experts have warned that physicians should consider reserving the current use of newer agents, such as fluoroquinolones, in order to preserve their activity for future patients. ${ }^{13,14}$ The recommendation to limit the use of newer drugs creates a tension for physicians caring for 
individual patients. Programs to reduce antibiotic use appeal to the community concerns of physicians at the cost of withholding treatments of potential benefit (both real and perceived) for individual patients.

The specific aims of this study were to identify physicians' antimicrobial drug choices for outpatients with community-acquired pneumonia, measure physicians' attitudes toward the balance between their public and individual patient health responsibilities, and determine the relationships between prescribing patterns and attitudes. We were specifically interested in the relative preference for prescribing a newer fluoroquinolone (levofloxacin) with limited current resistance relative to an older macrolide (erythromycin) with increasing drug resistance, ${ }^{15}$ even though it was still included as one of the first-line recommendations of most CAP guidelines available at the time. ${ }^{16,17}$ The former represents a drug with greater potential current patient benefit and the latter represents a drug with greater potential societal benefit in the sense that its use prevents overuse of newer drugs and thus delays emergence of resistance to these newer drugs. A secondary aim was to compare the patterns and attitudes of generalists and infectious diseases (ID) specialists, since the former make most of the empiric treatment decisions for patients with pneumonia but the latter often develop local and national guidelines.

\section{METHODS}

This study was a cross-sectional mailed survey of U.S. general internists, family practitioners, and infectious diseases specialists. The survey was conducted from April 2000 through June 2000.

\section{Questionnaire Development}

Based on prior work in eliciting provider treatment preferences, we utilized a series of rank order tasks to measure providers' relative preferences for different antimicrobial drug therapies in the treatment of patients with CAP. ${ }^{18}$ Rank order tasks provide greater distribution in respondent choices compared to simpler rating tasks for each choice, and when aggregated across a survey population can be used to generate relative ratings of these choices. Our primary response task was a ranking of 10 antimicrobial drug choices in the outpatient treatment of an otherwise uncomplicated 55-year-old male patient with CAP. The patient in the scenario had no comorbidities or drug allergies and was described as well enough to be treated as an outpatient. The 10 drugs were chosen to represent the major oral therapeutic drug classes available for the treatment of community-acquired pneumonia, including both older and newer agents: aminopenicillins (amoxicillin), extended spectrum aminopenicillins (amoxicillin/clavulanate), second-generation cephalosporins (cefuroxime axetil), third-generation cephalosporins (cefpodoxime proxetil), older macrolides (erythromycin), newer macrolides (azithromycin), older fluoroquinolones (ciprofloxacin), newer fluoroquinolones (levofloxacin), tetracyclines (doxycycline), and sulfa compounds (trimethoprim-sulfamethoxazole). Respondents were provided with both generic and brand names for each agent. At the time of the survey, the recommended drugs in the Infectious Diseases Society of America (IDSA) Guideline (1998) were macrolides (azithromycin, erythromycin, or clarithromycin), fluoroquinolones (levofloxacin, sparfloxacin, grepafloxacin, or trovafloxacin) or doxycycline. In general, the guideline recommended antibiotics in no particular order unless $H$. influenzae was suspected, where clarithromycin or azithromycin were preferred over erythromycin, or if penicillin-resistant $S$. pneumoniae was suspected, where a fluoroquinolone was recommended. Although we included many drug choices, we were specifically interested in the ranking of levofloxacin, a newer agent with limited current resistance relative to erythromycin, an older agent with increasing drug resistance, ${ }^{15}$ but still a first-line recommendation of most CAP guidelines.

A second rank task was developed to elicit the relative importance placed by physicians in determining their antimicrobial drug selections in this setting. Based on a series of one-on-one interviews and small-group discussions with generalists and ID specialists, we identified 7 basic determinants of drug prescribing: (1) potential side effects of the drug, (2) cost to patients, (3) ease of drug use, (4) efficacy of the drug in treating community-acquired pneumonia, (5) previous experience and knowledge about the drug, (6) severity of the illness, and (7) risk of the drug contributing to the problem of antibiotic resistance. Respondents were asked to rank order these 7 items in describing their antibiotic selection decisions for the preceding task.

Finally, based on our physician interviews, we created a series of attitude statements describing potential influences on antibiotic prescribing decisions. A total of 13 items were included in the questionnaire. For each item, respondents indicated their endorsement with a 5-point scale ranging from strongly disagree to strongly agree.

The survey was piloted with a small focus group of physicians and also mailed to an initial pilot sample of physicians to ensure the comprehensibility of the questions and response tasks.

\section{Subjects}

We randomly sampled 800 generalists (400 general internists and 400 family practitioners) and 800 infectious diseases specialists from the AMA Masterfile of U.S. physicians. The AMA Masterfile is the most comprehensive listing of U.S. physicians and includes non-AMA members. We excluded physicians without patient care activities as a primary activity.

Our survey design included an initial mailing with 2 follow-up mailings to nonrespondents. The initial mailing 
included a $\$ 5$ incentive. ${ }^{19}$ The third mailing was conducted with U.S. priority mail service.

\section{Analysis}

For the analysis of ranked data, we created adjusted ranked data for each antimicrobial drug choice, allowing for ties but reassigning subsequent choices to account for the total number of preceding choices. Missing values were assigned the lowest unassigned ranks. Using the adjusted rank data, we calculated the proportion of respondents who listed each antibiotic as their first choice. We also calculated the mean rank for each choice. A similar approach was used to analyze the ranked lists of factors influencing the selection of antibiotics in the preceding scenario.

Rank positions alone do not provide a sense of the relative distance between ranked items or whether different rank positions are statistically different from each other. However, a recently developed statistical technique, called exploded logit modeling, was designed to help analyze rank ordered data. The technique, based on proportional hazard modeling, compares the rank lists of the entire sample to calculate a relative risk of ranking any antibiotic higher than an arbitrarily chosen reference antibiotic. It thus allows one to calculate the relative preference for and statistical significance of one choice over another. In addition, because it is a multivariate technique, it allows one to analyze whether other factors, such as physician characteristics or attitudes, are significantly related to the ranked order of the antibiotic choices. ${ }^{20,21}$ We used erythromycin as the reference choice because it is one of the best-studied treatments for CAP and remained a first-line agent in guidelines available at the time of the study ${ }^{16,17}$ even though other, newer agents might have theoretical benefits for individual patients.

For the analysis of provider attitudes and beliefs regarding antimicrobial drug resistance, we report the distribution of ratings of each attitude, collapsing the strongly agree and agree categories into a single agree category and the strongly disagree and disagree categories into a single disagree category. Ratings of the middle category, "neutral" were omitted only for the purposes of data presentation. However, for the purposes of statistical comparisons, we compared the distributions of the entire 5-point rating scales between ID specialists and generalists with the nonparametric Wilcoxon 2-sample test.

Finally, we tested associations between the expressed attitudes of physicians and their self-reported patterns of antimicrobial drug selection in the pneumonia scenario. We were specifically interested in whether any particular attitudes were associated with the relative preference for levofloxacin compared to erythromycin. While it would have been possible to test each attitude question separately, we decided a priori to reduce the number of possible comparisons by identifying appropriate groupings of attitude statements and to create summary measures for each group of statements and test the association between these summary measures and the antibiotic choices. Principal components analysis was used to identify underlying groupings, or domains. Responses to all 13 attitude statements were analyzed and 4 domains were defined by grouping all items with a greater than 0.50 loading on factors identified in the rotated structure. We then created 4 domain scores by summing the responses to each statement within each of the 4 domains. These domain scores were dichotomized along the median value for the sample and separately entered as covariates in the exploded logit model, as previously described.$^{20}$ The results of this analysis tested the null hypothesis that there was no relationship between a higher value for each attitude domain and a relative preference for levofloxacin relative to erythromycin.

\section{RESULTS}

Out of the 1,600 questionnaires mailed, 21 were returned because of incorrect addresses or physician death and 829 physicians (53\%) responded to the survey. Four hundred responders were generalists (194 general internists and 206 family practitioners) and 429 were infectious diseases specialists. The mean age of the responders was 47 years, $73 \%$ were male, $88 \%$ were office based, and $19 \%$ were in solo practice. There were no statistically significant differences between responders and nonresponders in terms of clinical specialty, gender, age or geographic region, as recorded in the AMA Masterfile. However, responders were less likely to be office based and in solo practice compared to nonresponders (Table 1).

\section{Antibiotic Choices}

Overall, 33\% of respondents ranked azithromycin as their first antibiotic choice, $26 \%$ of respondents ranked levofloxacin as their first choice, and 10\% ranked amoxicillin as their first choice. Among generalists, 33\% ranked azithromycin first, 19\% ranked levofloxacin first, and 12\% ranked erythromycin first. Among infectious diseases specialists, 33\% ranked azithromycin first, 31\% ranked levofloxacin first, and 9\% ranked cefuroxime first. Table 2 displays the mean rankings for each of the 10 antibiotic drug choices. Overall, the top 3 drug choices were azithromycin, levofloxacin, and amoxicillin/clavulanate; among infectious diseases specialists the top 3 choices were azithromycin, levofloxacin, and cefuroxime axetil; and among generalists the top 3 choices were azithromycin, amoxicillin/clavulanate, and erythromycin.

The exploded logit model provides estimates of relative ratings for each antibiotic drug choice. For example, compared to erythromycin, azithromycin was 3.4 times more preferred as the first line treatment. Levofloxacin, amoxicillin/clavulanate and cefuroxime axetil were the next 3 favored drugs (relative preferences vs erythromycin 1.6, 1.6, 1.4, respectively), and all were preferred significantly 
Table 1. Characteristics of Survey Respondents and Nonrespondents

\begin{tabular}{|c|c|c|c|}
\hline Characteristic & $\begin{array}{l}\text { Responders, } \\
n=829(\%)\end{array}$ & $\begin{array}{c}\text { Nonresponders, } \\
n=750(\%)\end{array}$ & $P$ Value* \\
\hline Medical specialty & & & .27 \\
\hline Generalist & $400(48)$ & $388(52)$ & \\
\hline Internal medicine & $194(23)$ & $200(27)$ & \\
\hline Family practice & $206(25)$ & $188(25)$ & \\
\hline Infectious diseases & $429(52)$ & $362(48)$ & \\
\hline Male gender & $605(73)$ & $539(72)$ & .62 \\
\hline \multicolumn{4}{|l|}{ Age, y } \\
\hline$\leq 40$ & $245(30)$ & $200(27)$ & .42 \\
\hline $41-59$ & $481(58)$ & $457(61)$ & \\
\hline$\geq 60$ & $102(11)$ & $93(12)$ & \\
\hline U.S. region & & & .36 \\
\hline East & $217(27)$ & $186(25)$ & \\
\hline Midwest & $188(23)$ & $150(20)$ & \\
\hline South & $250(31)$ & $238(32)$ & \\
\hline West & $156(19)$ & $149(20)$ & \\
\hline \multicolumn{4}{|l|}{ Practice setting } \\
\hline Office-based & $729(88)$ & $685(91)$ & .03 \\
\hline Hospital staff & $100(12)$ & $65(9)$ & \\
\hline Type of practice & & & .002 \\
\hline Solo & 154 (19) & $173(22)$ & \\
\hline Group & $243(29)$ & $187(23)$ & \\
\hline Staff model & $106(13)$ & $70(10)$ & \\
\hline Not classified & $276(33)$ & $302(40)$ & \\
\hline
\end{tabular}

* $X^{2}$ test statistic for difference in distribution between responders and nonresponders.

more than erythromycin. All remaining drugs were significantly less preferred than erythromycin.

Physician specialty was significantly associated with the relative preferences for antibiotics in the treatment of community-acquired pneumonia $(P<.0001$ for the overall effect of physician specialty in determining relative antibiotic preferences from the exploded logit model). In particular, infectious diseases specialists significantly preferred azithromycin, levofloxacin, amoxicillin/clavulanate and cefuroxime axetil over erythromycin. In contrast, generalists preferred only azithromycin over erythromycin. Generalists' preferences for levofloxacin, amoxicillinclavulanate, and cefuroxime axetil were statistically indistinguishable from erythromycin. Erythromycin was significantly preferred over the remaining drugs, most of which, with the exception of doxycycline, were not recommended by the available CAP guidelines. ${ }^{16,17}$

Among generalists, the ranked preferences of general internists and family practitioners were very similar.

The top 3 factors influencing drug choice were efficacy of the drug in treating CAP, severity of the illness, and previous experience and knowledge about the drug (Table 3). The risk of contributing to the problem of antibiotic resistance was ranked lowest, overall and by generalists, and second lowest by ID specialists.

\section{Attitudes}

Principal components analysis of the 13 attitude statements identified 4 underlying domains regarding the problem of antibiotic resistance and its relationship to antibiotic drug prescribing (Table 4). Each of the 13 statements had a loading of $>0.50$ on 1 of the 4 domains and no item had a loading of $>0.50$ on more than 1 domain; i.e., the loading structure defined 4 mutually exclusive domains.

The first domain reflects physician concern over the societal impact of drug resistance. All statements within this domain were endorsed by the majority of infectious diseases specialists and generalists.

The second domain reflects the belief that the responsibility for facing the problem of antibiotic resistance does not reside within each individual physician because the

Table 2. Antibiotic Preferences for Patients with CAP

\begin{tabular}{|c|c|c|c|c|c|c|}
\hline Drug & \multicolumn{2}{|c|}{ All Physicians } & \multicolumn{2}{|c|}{ ID Specialists } & \multicolumn{2}{|c|}{ Generalists } \\
\hline Levofloxacin & 4.1 & $1.6^{\ddagger}$ & 3.5 & $3.1^{\ddagger}$ & 4.8 & 0.8 \\
\hline Amoxicillin/clavulanate & 4.6 & $1.6^{\ddagger}$ & 4.8 & $1.9^{\ddagger}$ & 4.3 & 1.2 \\
\hline Cefuroxime axetil & 4.8 & $1.4^{\ddagger}$ & 4.5 & $2.2^{\ddagger}$ & 5.2 & 0.8 \\
\hline Amoxicillin & 6.2 & $0.6^{\ddagger}$ & 6.5 & 0.8 & 5.9 & $0.5^{\ddagger}$ \\
\hline Cefpodoxime proxetil & 6.7 & $0.6^{\ddagger}$ & 6.4 & 0.9 & 7.0 & $0.4^{\ddagger}$ \\
\hline Trimethoprim-sulfamethoxazole & 6.8 & $0.6^{\ddagger}$ & 6.7 & 0.8 & 6.8 & $0.4^{\ddagger}$ \\
\hline Ciprofloxacin & 7.4 & $0.4^{\ddagger}$ & 8.0 & $0.4^{\ddagger}$ & 6.7 & $0.4^{\ddagger}$ \\
\hline
\end{tabular}

* Physicians ranked their choices of antibiotic from $1=$ most preferred to $10=$ least preferred.

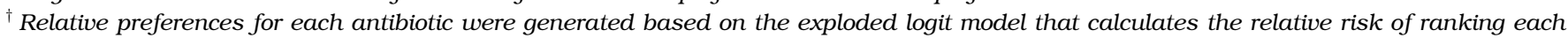
antibiotic lower (i.e., more preferred) compared to the reference choice, erythromycin.

${ }^{\ddagger} \mathrm{P}<.0001$ compared to reference category.

CAP, community-acquired pneumonia; ID, infectious diseases. 
Table 3. Importance of Factors Influencing the Selection of Antibiotics for Patients with CAP

\begin{tabular}{|c|c|c|c|}
\hline Factor & $\begin{array}{c}\text { Mean Rank } \\
\text { All Physicians }\end{array}$ & $\begin{array}{l}\text { Mean Rank by } \\
\text { ID Specialists }\end{array}$ & $\begin{array}{c}\text { Mean Rank } \\
\text { by Generalists }\end{array}$ \\
\hline Efficacy of the drug in treating community-acquired pneumonia & 1.6 & 1.5 & 1.8 \\
\hline Previous experience and knowledge about the drug & 4.1 & 4.3 & 4.0 \\
\hline Potential side effects & 4.4 & 4.4 & 4.4 \\
\hline Ease of use & 4.6 & 4.5 & 4.8 \\
\hline
\end{tabular}

CAP, community-acquired pneumonia; ID, infectious diseases.

physician is obligated to consider only individual patients' needs with each prescribing decision (statements 1 and 3), or because the problem is not clinically relevant due to the pace of new drug development (statement 2), or because it is not the prescribing but the taking of antibiotics that causes the problem (statement 4). For each item in the domain, infectious diseases specialists were significantly more likely to disagree compared to generalists. For example, $64 \%$ of infectious diseases specialists compared to $49 \%$ of generalists disagreed with the statement that new drug development would keep pace with the problem of antibiotic resistance (thus relieving physicians from that responsibility).

The third domain is related to the first domain in that it reflects concern over the societal impact of drug resistance but more specifically focuses on the ability of physicians to balance societal and patient needs in prescribing antibiotics. The majority of respondents agreed with both

Table 4. Attitudes and Beliefs of Providers Toward Antibiotic Drug Prescribing and Antibiotic Resistance

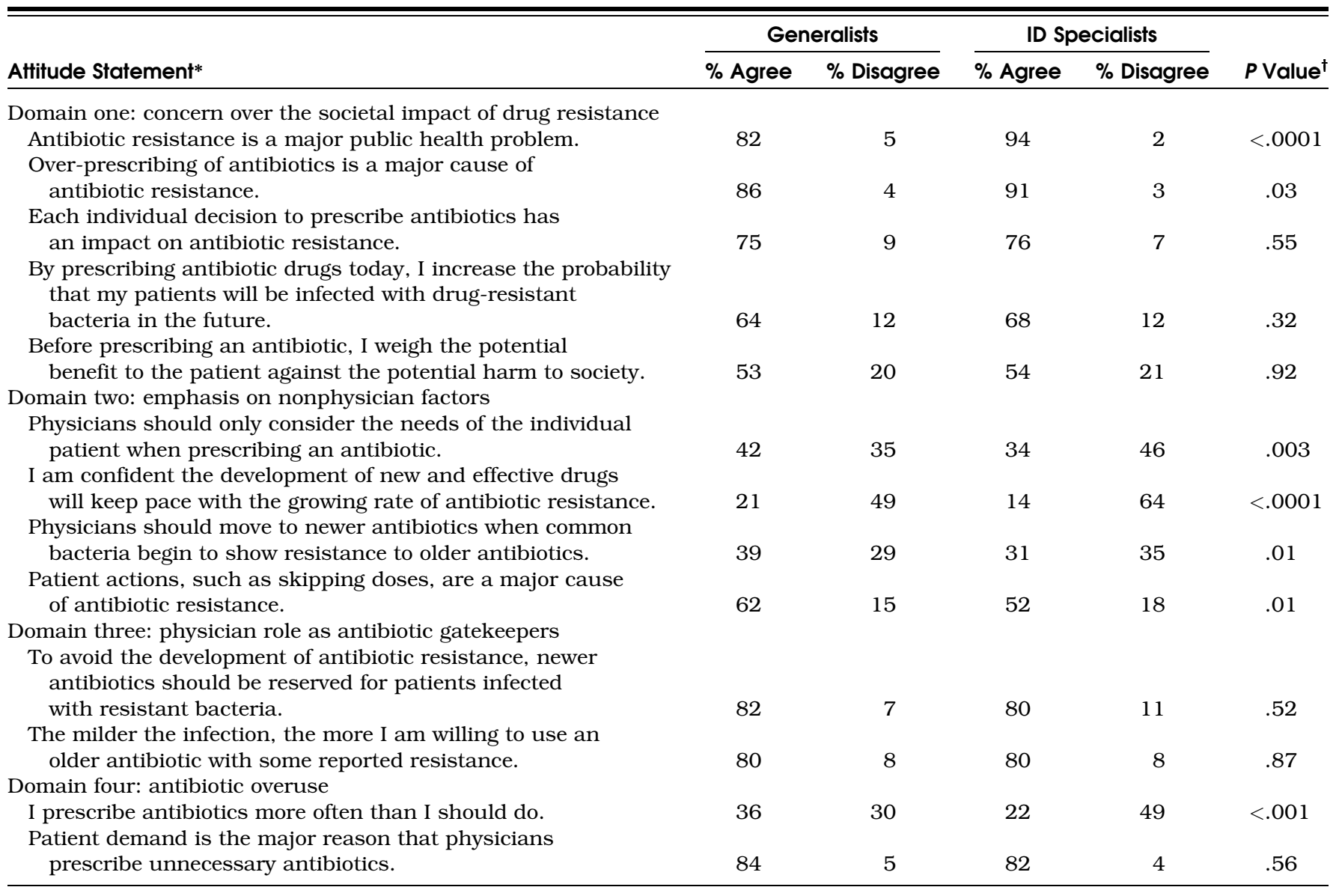

* Domains were identified based on Principal Components Analysis, combining all items with >0.5 loadings on the rotated factor patterns.

$\dagger$ Two-sample Wilcoxon Test comparing ratings of generalists and ID specialists.

ID, infectious deseases. 
items, and there were no significant differences between generalists and infectious diseases specialists in this domain.

The fourth domain reflects the problem of antibiotic overuse. The majority of respondents agreed that patient demand was a major reason for antibiotic overprescribing, while only a minority of respondents agreed that they personally prescribe antibiotics more than they should. Generalists were more likely than infectious diseases specialists to agree that they prescribe antibiotics more often than they should ( $36 \%$ vs $22 \%$; $P=.001$ ).

Among generalists, the expressed attitudes were similar between general internists and family practitioners.

\section{Associations Between Attitudes and Antimicrobial Choices}

Incorporating the scores for each attitude domain into the exploded logit model revealed that 2 of the 4 attitude domains were significantly associated with the antibiotic ranking patterns. For the domain reflecting balance between individual and societal needs (Domain 3), higher agreement with the importance of societal interests was associated with a significant reduction in the magnitude of the preference for levofloxacin relative to erythromycin $(P<.0001)$. For the domain reflecting concern over patient needs (Domain 2), higher agreement with the importance of the individual patient was associated with a significant increase in the magnitude of the preference for levofloxacin relative to erythromycin $(P<.001)$.

\section{DISCUSSION}

While guidelines for the treatment of CAP provide a range of appropriate antibiotic choices, there has been increasing evidence that the specific choices are not equivalent in terms of their risk of accelerating the rate of emerging antibiotic resistance. Both the use of newer fluoroquinolones and the use of newer macrolides have been implicated as risk factors for recent increases in rates of $S$. pneumoniae resistance to these agents. ${ }^{1,22}$ These results suggest that in order to combat rising antibiotic resistance, patterns of antibiotic prescribing should consider not only patient issues of efficacy, cost, and tolerability but also public health issues of future drug resistance. Several findings from this study suggest that physicians are unlikely to consider this public health issue alongside the individual patient issues that drive antibiotic choices.

First, both generalists and infectious diseases specialists preferred newer, broader antibiotics (azithromycin, levofloxacin, amoxicillin/clavulanate) compared to older agents (erythromycin, doxycycline) for the treatment of outpatients with CAP even though the most current available guideline at the time did not recommend the newer agents over the older agents for typical cases. ${ }^{16}$ Second, although most physicians acknowledged that antimicrobial resistance is a major public health problem caused by physicians' antimicrobial drug choices, the issue was consistently ranked lower than patient-specific issues, such as efficacy, cost, and tolerability, in determining specific drug choices.

Emerging antimicrobial resistance is a public health concern, but these findings suggest that individual physicians underweight their responsibilities to this social goal. While the issue of antibiotic resistance at a societal level was the only factor among the choices in our question that did not directly relate to individual patient care, physicians consistently ranked this factor lowest in importance. The guidelines available at the time of this survey endorsed macrolides, tetracyclines, and in some settings $\beta$-lactams as adequate therapy for the majority of patients, reserving newer fluoroquinolones for patients at increased risk of drug-resistant pneumococcal infections. ${ }^{14,16,17}$ In contrast, in our study, erythromycin, amoxicillin, and doxycycline were consistently ranked lower than newer, broader agents including a newer fluoroquinolone, levofloxacin, and a newer macrolide, azithromycin.

Few studies have evaluated the impact of national guidelines on the pattern of physician use of antibiotics, particularly for community-acquired pneumonia. To some degree, this may reflect the existence of multiple guidelines in this area, often with conflicting recommendations. In one prospective study of patients with community-acquired pneumonia treated at 5 different outpatient sites in 3 distinct geographic regions, a total of 23 different antibiotic regimens were prescribed. Significant variation in prescribing rates occurred for 17 of these antibiotic regimens across the different clinical sites. ${ }^{23}$ Such variation suggests that local factors such as formulary restrictions and opinion leaders have a strong impact on the actual patterns of antibiotic prescribing. On the other hand, there is little evidence to suggest that national guidelines, particularly when they emphasize societal concerns, have much impact on individual antibiotic prescribing decisions. For example, in the same study, only $46 \%$ of outpatients were prescribed antibiotic regimens that were consistent with the American Thoracic Society treatment guidelines available at the time of the study. ${ }^{24}$

One important difference in the antibiotic rankings of generalists versus infectious diseases specialists was that while both groups were statistically more likely to prefer azithromycin over erythromycin, infectious diseases specialists were also statistically more likely to prefer levofloxacin over erythromycin, while generalists were relatively indifferent. One possible explanation for this finding is that infectious diseases specialists adopt newer therapies more rapidly than generalists because they learn about these therapies more rapidly. However, levofloxacin had been FDA approved for the treatment of CAP over 3 years prior to this survey and it was mentioned by name in the IDSA guidelines published 2 years before this survey. Given that both groups ranked most patient factors as more important than societal factors in influencing their prescribing decisions, it is likely that differences in the preferences for drugs between these 2 
groups are driven more by issues of perceived efficacy, cost, and tolerability and less by differences in concern over the impact on future rates of resistance.

This study has limitations. First, these study results are based on self-reported actions and not observed practice patterns in clinical settings. Second, although our response rate of $53 \%$ is consistent with norms for physician mail surveys, ${ }^{25,26}$ our results may be subject to nonresponse bias. Third, this study provides a description of decisions and attitudes at only one point in time. Moreover, we do not know whether respondents were familiar with specific guidelines available at that time and to what degree the guidelines influenced their prescribing decisions. Since the completion of this survey, new guidelines from both the IDSA and the Canadian Infectious Diseases Society were released and further emphasize the importance of weighing the current and future risks of antimicrobial drug resistance in the selection of drugs for the treatment of patients with pneumonia. ${ }^{3,27}$ Finally, another factor that is important in interpreting variation in physician patterns of antibiotic use is the local rates of antibiotic resistance, particularly among $S$. pneumoniae, and physician awareness of these rates. For example, physicians who practice in areas with high rates of penicillin and macrolide resistance may be much more likely to prefer fluoroquinolones for the treatment of CAP. However, we did not collect data on the local rates of drug resistance for each physician's practice.

In summary, neither generalists nor infectious diseases specialists emphasize the relative societal risks of antimicrobial drug selection in their treatment decisions for patients with community-acquired pneumonia. Instead, they emphasize providing the newest and best treatments for each individual patient even though this approach may not be supported by current guidelines or public health policy. Whenever antibiotic prescribing decisions create a tension between the interests of an individual patient and the broader public, individual physician decisions are likely to disfavor public interests. Thus, guideline recommendations and educational programs alone are unlikely to achieve ideal prescribing from a public health perspective, and future efforts to optimize antibiotic choices may need more force. In the hospital setting, it is increasingly common for antimicrobial management programs to rely on order entry systems, formulary restrictions, and peer leaders to promote specific patterns of antibiotic use to combat rising resistance within the hospital. ${ }^{28}$ Such antibiotic regulation may be an increasingly necessary feature of outpatient care as well.

We thank Christine Hardy, MS (Center for Clinical Epidemiology and Biostatistics) for her valuable contributions in the analysis of the ranked data.

This work was supported by the Robert Wood Johnson Foundzation through a Generalist Physician Faculty Scholar Award to Dr. Metlay. Dr. Metlay is also supported by the
Department of Veterans Affairs through a Research Career Development Award.

\section{REFERENCES}

1. Levy SB. Confronting multidrug resistance. A role for each of us. JAMA. 1993;269:1840-2.

2. Cohen ML. Epidemiology of drug resistance: implications for a post-antimicrobial era. Science. 1992;257:1050-5.

3. Bartlett JG, Dowell SF, Mandell LA, File TM Jr, Musher DM, Fine MJ. Practice guidelines for the management of communityacquired pneumonia in adults. Infectious Diseases Society of America. Clin Infect Dis. 2000;31:347-82.

4. Warren JW, Abrutyn E, Hebel JR, Johnson JR, Schaeffer AJ, Stamm WE. Guidelines for antimicrobial treatment of uncomplicated acute bacterial cystitis and acute pyelonephritis in women. Infectious Diseases Society of America (IDSA). Clin Infect Dis. 1999;29:745-58.

5. Schwartz B, Bell DM, Hughes JM. Preventing the emergence of antimicrobial resistance. A call for action by clinicians, public health officials, and patients. JAMA. 1997;278:944-5.

6. Schwartz B. Preventing the spread of antimicrobial resistance among bacterial respiratory pathogens in industrialized countries: the case for judicious antimicrobial use. Clin Infect Dis. 1999; 28:211-3.

7. Gonzales R, Bartlett JG, Besser RE, et al. Principles of appropriate antibiotic use for treatment of acute respiratory tract infections in adults: background, specific aims, and methods. Ann Intern Med. $2001 ; 134: 479-86$.

8. Low DE, Scheld WM. Strategies for stemming the tide of antimicrobial resistance. [editorial; comment]. JAMA. 1998;279: 394-5.

9. Seppala H, Klaukka T, Vuopio-Varkila J, et al. The effect of changes in the consumption of macrolide antibiotics on erythromycin resistance in group A streptococci in Finland. Finnish Study Group for Antimicrobial Resistance. N Engl J Med. 1997;337: 441-6.

10. Arason VA, Kristinsson KG, Sigurdsson JA, Stefansdottir G, Molstad S, Gudmundsson S. Do antimicrobials increase the carriage rate of penicillin-resistant pneumococci in children? Cross sectional prevalence study. BMJ. 1996;313:387-91.

11. Chen DK, McGeer A, de Azavedo JC, Low DE. Decreased susceptibility of Streptococcus pneumoniae to fluoroquinolones in Canada. Canadian Bacterial Surveillance Network. N Engl J Med. 1999;341:233-9.

12. Niederman MS, Mandell LA, Anzueto A, et al. Guidelines for the management of adults with community-acquired pneumonia. Diagnosis, assessment of severity, antimicrobial therapy, and prevention. Am J Respir Crit Care Med. 2001;163:1730-54.

13. Hooper DC. Expanding uses of fluoroquinolones: opportunities and challenges. Ann Intern Med. 1998;129:908-10.

14. Heffelfinger JD, Dowell SF, Jorgensen JH, et al. Management of community-acquired pneumonia in the era of pneumococcal resistance: a report from the Drug-Resistant Streptococcus pneumoniae Therapeutic Working Group. Arch Intern Med. 2000;160: 1399-408.

15. Whitney CG, Farley MM, Hadler J, et al. Increasing prevalence of multidrug-resistant Streptococcus pneumoniae in the United States. N Engl J Med. 2000;343:1917-24.

16. Bartlett JG, Breiman RF, Mandell LA, File TM Jr. Communityacquired pneumonia in adults: guidelines for management. The Infectious Diseases Society of America. Clin Infect Dis. 1998;26: 811-38.

17. Niederman MS, Bass JB Jr., Campbell GD, et al. Guidelines for the initial management of adults with community-acquired pneumonia: diagnosis, assessment of severity, and initial antimicrobial therapy. American Thoracic Society. Medical Section of 
the American Lung Association. Am Rev Respir Dis. 1993;148: 1418-26.

18. Christakis NA, Asch DA. Medical specialists prefer to withdraw familiar technologies when discontinuing life support. J Gen Intern Med. 1995; 10:491-4.

19. Asch DA, Christakis NA, Ubel PA. Conducting physician mail surveys on a limited budget. A randomized trial comparing $\$ 2$ bill versus \$5 bill incentives. Med Care. 1998;36:95-9.

20. Allison P, Christakis N. Logit models for sets of ranked items. Sociolog Methodol. 1994;24:199-228.

21. Asch DA, Christakis NA. Why do physicians prefer to withdraw some forms of life support over others? Intrinsic attributes of life-sustaining treatments are associated with physicians' preferences. Med Care. 1996;34:103-11.

22. Baquero F. Evolving resistance patterns of Streptococcus pneumoniae: a link with long-acting macrolide consumption? J Chemother. 1999;11:35S-43S.

23. Gilbert K, Gleason PP, Singer DE, et al. Variation in antimicrobial use and cost in more than 2,000 patients with communityacquired pneumonia. Am J Med. 1998;104:17-27.

24. Gleason PP, Kapoor WN, Stone RA, et al. Medical outcomes and antimicrobial costs with the use of the American Thoracic Society guidelines for outpatients with community-acquired pneumonia. JAMA. 1997;278:32-9.

25. Asch DA, Jedrziewski MK, Christakis NA. Response rates to mail surveys published in medical journals. J Clin Epidemiol. 1997;50: 1129-36.

26. Cummings SM, Savitz LA, Konrad TR. Reported response rates to mailed physician questionnaires. Health Serv Res. 2001;35: 1347-55.

27. Mandell LA, Marrie TJ, Grossman RF, Chow AW, Hyland RH. Canadian guidelines for the initial management of communityacquired pneumonia: an evidence-based update by the Canadian Infectious Diseases Society and the Canadian Thoracic Society. The Canadian Community-Acquired Pneumonia Working Group. Clin Infect Dis. 2000;31:383-421.

28. Shlaes DM, Gerding DN, John JF Jr., et al. Society for Healthcare Epidemiology of America and Infectious Diseases Society of America Joint Committee on the Prevention of Antimicrobial Resistance: guidelines for the prevention of antimicrobial resistance in hospitals. Clin Infect Dis. 1997;25: 584-99.

\section{JOURNAL OF GENERAL INTERNAL MEDICINE SUBSCRIBERS Do we have your new address?}

Send us your new address three months before it becomes effective, so we will have time to get it into our computer system and ensure that your copies of JGIM continue to arrive uninterrupted. Send your old mailing label, your new address with zip code, the effective date of your new address, and your current telephone number.

\author{
Nonmember subscribers notify: \\ Rochelle Belanger \\ Blackwell Science, Inc. \\ Commerce Place, 350 Main St. \\ Malden, MA 02148
}

SGIM members notify:

Katrese Phelps

Society of General Internal Medicine 2501 M Street, NW, Suite 575

Washington, DC 20037 